\title{
MYANMAR: EMPOWERING HEALTH WORKERS \& LEVERAGING DIGITAL TECHNOLOGY TO IMPROVE ACCESS TO MENTAL HEALTH CARE IN HLAING THAR YAR TOWNSHIP
}

Khin Maung Gyee

Project Manager, Myanmar Medical Association, Yangon, Myanmar.

\section{Objectives}

This pilot project conducted in Hlaing Thar Yar, a highly populated township of the Western part of Yangon, aims to reduce the treatment gap for mental disorders (especially depression, psychosis and epilepsy) by $20 \%$. (ie, to reduce the proportion of patients who are not treated by $20 \%$ )

\section{Background and aims}

Despite an estimated $8 \%$ prevalence for mental disorders in Myanmar $^{1}$, the treatment gap is approximately $90 \%{ }^{1}$. This is due to the scarcity of healthcare workers specialized in mental health $(1.18 / 100,000 \text { people })^{2}$, the insufficient mental health training of GPs ( $1 \%$ total GP training time $)^{3}$, combined with false beliefs and prejudices within the community.

\section{Materials and methods}

The objectives of this project are to upskill and empower existing township human resources:

-75 CHWs to raise awareness, identify people with mental disorders and refer to GPs, support patients and families;

-90 GPs (and other 1ry HCPs), to diagnose and manage patients.

Low-cost digital technology (smartphones \& electronic tablets) helps identify people with mental disorders (interactive screening questionnaires on CHWs' smartphones), refer from CHWs to GPs (data recorded by CHWs available to GPs), and assists in the diagnosis and management of patients (WHO mhGAP intervention guide, e-medical records etc. available through GPs' tablets).

After a 6-month preparation phase which included designing behaviour change communication (BCC) tools to be used by $\mathrm{CHWs}$, developing training materials, procurement of the smartphones \& tablets, development of the apps, as well as recruitment and training of the CHWs \& primary HCPs, the 2-year field implementation phase could start in November 2017

\section{Interim Results}

The following key outputs and outcomes have been achieved so far (1 Aug 19):

$\approx 17,000$ people have attended information meetings held by CHWs

1,308 people have been screened by CHWs and referred to GPs
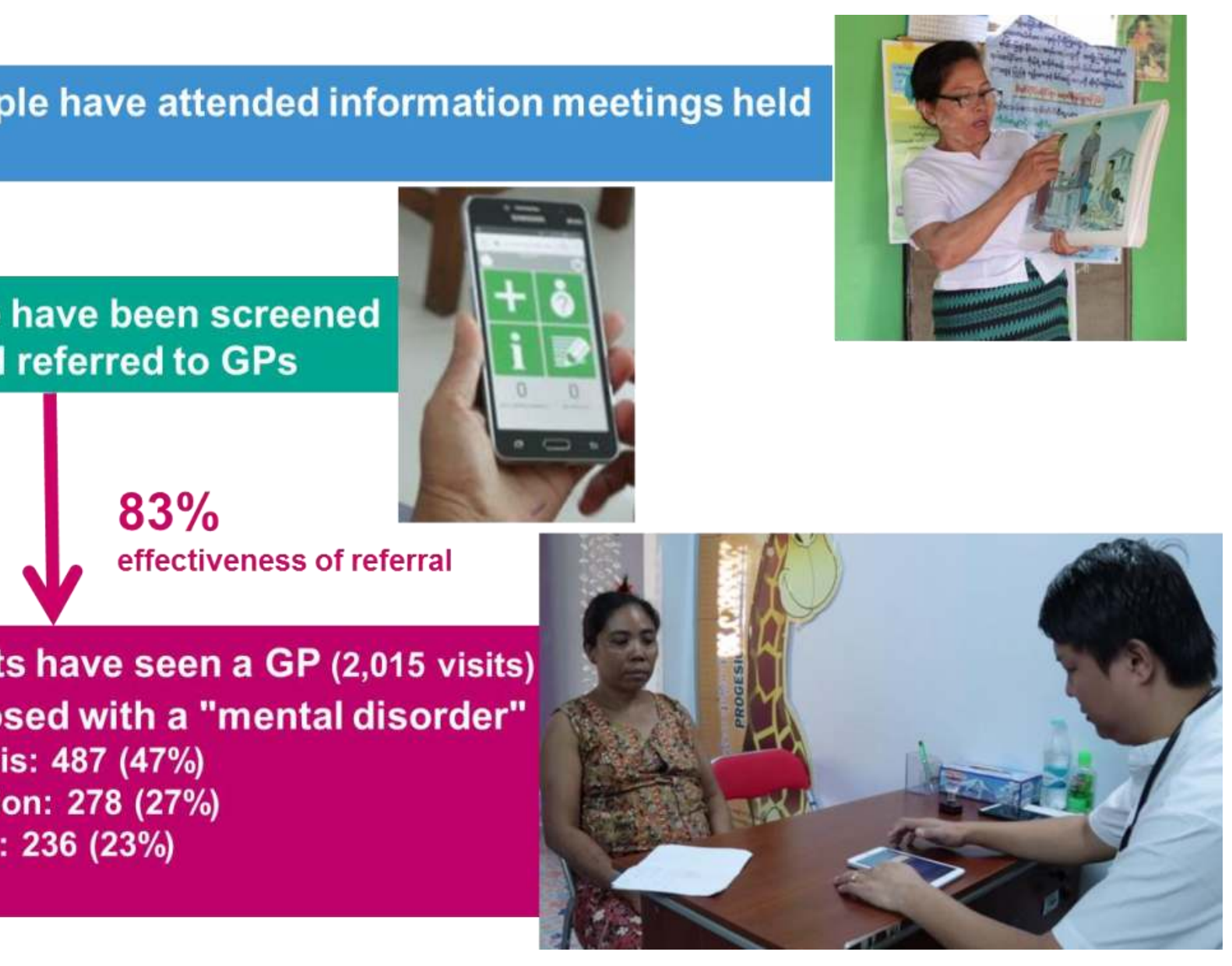

Ref: 1. Win Aung Myint et al. Myanmar; Routledge Handbook of Psychiatry in Asia. 2016 2. WHO - Mental Health Atlas 2017. 3.WHO-AIMS Report on Mental Health in Myanmar. 2006

Project funded by Sanofi Global Health Programs

Copyright @ 2019 Prof Khin Maung Gyee, Yangon, Myanmar
Effectiveness of CHW screening and referral

- $\mathbf{8 3} \%(1,090 / 1,308)$ of patients screened and referred by CHWs have seen a GP

- 92\% $(1,001 / 1,090)$ of patients seen by GPs have been diagnosed with one of the 3 targeted diseases.

- Overall $\mathbf{8 1} \%$ concordance between CHW screening and GP diagnosis

\begin{tabular}{|l|r|r|r|}
\hline \multicolumn{2}{|c|}{ Suspected cases by CHWs confirmed by GPs (number and \%) } \\
\hline & $\begin{array}{c}\text { Suspected } \\
\text { by CHW }\end{array}$ & $\begin{array}{c}\text { Diagnosed } \\
\text { by GP }\end{array}$ & $\begin{array}{c}\text { Concordance } \\
\%\end{array}$ \\
\hline Psychosis & 445 & 369 & $83 \%$ \\
\hline Depression & 311 & 220 & $71 \%$ \\
\hline Epilepsy & 225 & 202 & $90 \%$ \\
\hline
\end{tabular}

\section{Discussion - Key learnings}

\section{Strengths}

-The electronic referral system is working: data collected by $\mathrm{CHWs}$ on their smartphone is accessed by GPs on their tablet

-With appropriate training, CHWs can raise awareness and help identify people with mental disorders

-information meetings held by $\mathrm{CHWs}$ are leading to case findings

-interactive questionnaires on smartphone are assisting in screening for

psychosis, depression \& epilepsy

-CHW's advice, referral and follow-up are impactful:

$\mathbf{- 8 3 \%}$ of people $(1,090 / 1,308)$ referred by a CHW have actually seen a GP

-CHW screening seems accurate

$-92 \%$ of people who have seen a GP have been diagnosed with a "mental disorder"

$\mathbf{1 8 1 \%}$ concordance between CHW screening \& GP diagnosis

Very positive feedback from GPs:

-training, CHW, app \&tablet $\rightarrow$ perceived positively by $93-98 \%$ of GPs

$\checkmark 90 \%$ would like to see more patients referred by $\mathrm{CHWs}$

\section{Limitations}

-Recruitment (screening \& diagnosis) is slowing down

Cumulative number of positive screenings by $\mathrm{CHWs}$ and patients diagnosed by GPs

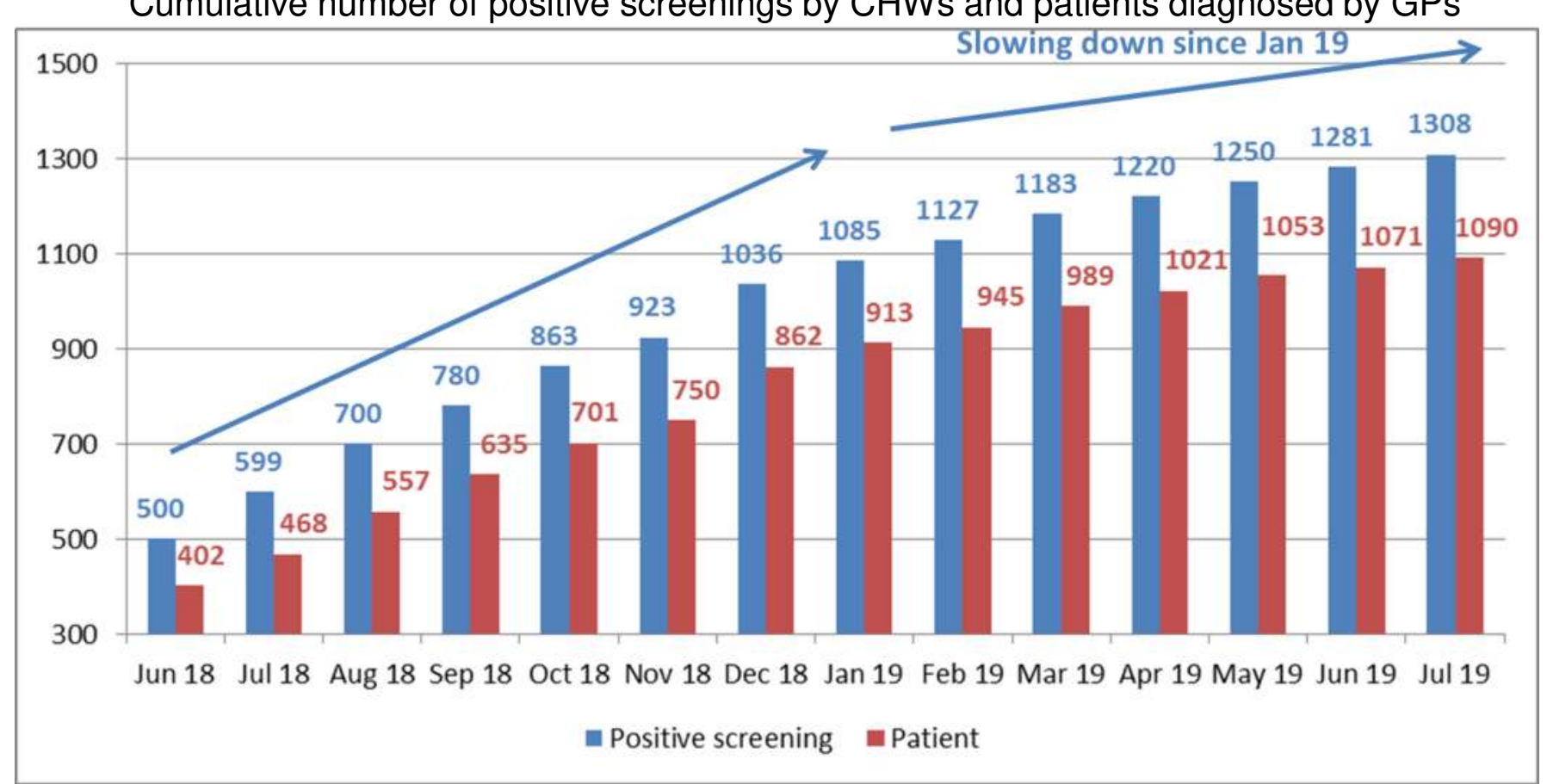

-High apparent drop-out rate (44\% between V2 \& V3) due to

- GPs failing to record data in system when seeing patients at follow-up visits

-Mobility of population

- Lack of subsidised treatment / Cost of Drugs

\section{Conclusions}

This 3 year project, launched in April 2017 by the Myanmar Medical Association, in partnership with the Myanmar Mental Health Society, the World Association of Social Psychiatry, the Universite Numérique Francophone Mondiale and Sanofi, has already shown shown some positive outcomes. Despite some drawbacks, the results so far suggest that CHWs, with appropriate training and tools, can play a very important role in identifying patients with mental disorders and referring them to GPs for treatment. 